\title{
Study on Experimental Leishmanicidal Activity and in silico of Cytochalasin B
}

\author{
André O. Feitosa, ${ }^{a}$ Fábio J. N. Ferreira, ${ }^{\circledR b}$ Heliton P. C. Brigido, ${ }^{b}$ Mirian L. C. Bastos, ${ }^{b}$ \\ Josiwander M. Carvalho, ${ }^{a}$ Agnaldo S. Carneiro, ${ }^{b}$ Maria F. Dolabela, ${ }^{b}$ \\ Patrícia S. B. Marinho ${ }^{a}$ and Andrey M. R. Marinho ${ }^{\circledR *, a}$
}

aPrograma de Pós-Graduação em Química, Instituto de Ciências Exatas e Naturais, Universidade Federal do Pará, 66075-900 Belém-PA, Brazil

${ }^{b}$ Faculdade de Fármacia, Universidade Federal do Pará, 66075-900 Belém-PA, Brazil

\begin{abstract}
Leishmaniasis is a neglected infectious disease caused by different species of the Leishmania parasite and is one of the major public health problems in developing countries. Despite the progress in fundamental knowledge about the Leishmania parasite, current therapy against leishmaniasis is still unsatisfactory due to limited efficacy, prolonged treatment, high cost and undesirable adverse effects. Thus, the research for new prototypes compounds of antileishmaniasis drugs remains a matter of current importance. The objective of this study was to evaluate the leishmanicidal activity of cytochalasin B, a natural compound isolated by our group in a previous study, against L. amazonensis, using experimental tests and computational simulation methodologies. The results of the biological evaluation showed that cytochalasin B has antileishmanial activity against the promastigote form of Leishmania amazonensis. These results are corroborated by the docking and molecular dynamics that showed that the activity occurs due to the inactivation of the trypanothione reductase enzyme (TryR).
\end{abstract}

Keywords: cytochalasin B, antileishmaniasis, molecular docking, molecular dynamics

\section{Introduction}

The search for new drugs for neglected human diseases has accelerated in the last decade. Neglected diseases are a set of infectious diseases differentiated by the high level of morbidity, however, with a low level of mortality. They seriously affect the quality of life and cause negative socio-economic impacts for the population of the poorest countries. ${ }^{1,2}$

Leishmaniasis is one of the major public health problems in developing countries. This disease is among the endemics considered a priority in the world, being cited in 98 countries and territories, affecting around 2 million people per year, with 350 million people being exposed to infection. ${ }^{3}$

In addition to the toxicity of antileishmaniasis drugs, another serious problem that makes the treatment of the disease difficult is the development of resistance by the parasite. ${ }^{4}$ Studies have shown isolated parasites resistant to antimonials. ${ }^{5,6} \mathrm{~A}$ modern approach in the search for new drugs is through the use of theoretical medicinal chemistry combined with the classical experimental

*e-mail: andrey@ufpa.br methods. Molecular modeling has as main objective to reproduce the real (experimental) behavior of molecules and molecular systems. ${ }^{7}$ Based on computational studies, the most promising compounds can be synthesized and tested, which greatly increases the chances of discovering a biologically active molecule that can be used as a drug. The technologies in silico, when compared to the in vitro and in vivo methods, are less expensive, faster, have a higher yield, have a greater reproducibility, and have the potential to reduce the use of animals, ${ }^{8}$ in addition to its important applications in the rational planning of potential bioactive compounds, particularly compounds with antileishmanial activity. ${ }^{9}$

In this study, the leishmanicidal activity of cytochalasin B (1) (Figure 1) was studied against a

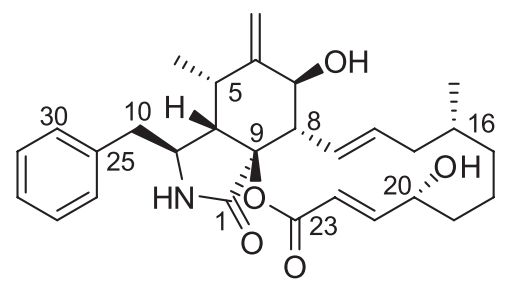

Figure 1. Structure of cytochalasin B (1). 
strain of L. amazonensis, as well as the application of the molecular docking technique and computational simulation through classic molecular dynamics (MD). In both assays, cytochalasin B (1) showed good activity.

\section{Experimental}

Isolation of cytochalasin B

The cytochalasin B used in this work was isolated from extracts of the fungal biomass of Aspergillus EJC04 obtained from the collection of Bauhinia guianensis isolates from the Laboratório de Bioensaios e Química de Microorganismos (LaBQuiM, Universidade Federal do Pará, Brazil). The cultivation of the microorganism, as well as the extraction of the extracts, isolation and identification of cytochalasin B (1) has been described in a previous work. ${ }^{10}$

\section{Species of Leishmania and cultivation}

In this study, promastigotes of Leishmania (L.) amazonensis, isolated from a patient from the municipality of Ulianópolis in the state of Pará, provided by Instituto Evandro Chagas (ICE, Ananindeua, Pará) under the record No. MHOM/BR/2009/M26361, were used. Promastigote forms of the species $L$. (L.) amazonensis were grown in Roswell Park Memorial Institute growth medium (RPMI 1640) and kept through weekly passages as described. $0.5 \mathrm{~mL}$ of promastigote suspension was transferred to cell culture bottles, each containing $5 \mathrm{~mL}$ of complete RPMI medium. Next, the inverted microscope culture was observed to verify the viability of the forms in RPMI medium. Cultivation of the parasite in complete RPMI medium was done at $26 \pm 1{ }^{\circ} \mathrm{C}$.

\section{Antipromastigote activity test}

Promastigotes forms of $L$. (L.) amazonensis obtained during the logarithmic growth phase were pooled by centrifugation in complete RPMI medium at 3,500 rpm for $10 \mathrm{~min}$. The precipitate was re-suspended in complete RPMI medium; the promastigotes were quantified in a Neubauer chamber and adjusted to a concentration corresponding to $5 \times 10^{6}$ parasites $\mathrm{mL}^{-1}$. The susceptibility test was performed in 96-well plates. The cytochalasin $\mathrm{B}$ was tested in triplicate in a concentration gradient (200 to $3.125 \mu \mathrm{g} \mathrm{mL}^{-1}$ ). The negative control contained only parasites and the incubation medium, and the positive control was made with amphotericin B (25 to $\left.0.3906 \mu \mathrm{g} \mathrm{mL}{ }^{-1}\right)$. After $24 \mathrm{~h}$ of incubation at $26^{\circ} \mathrm{C}, 10 \mu \mathrm{L}$ of tetrazolium salt $\left(5 \mathrm{mg} \mathrm{mL}^{-1}\right)$ were added, and the parasites were quantified in enzyme-linked immunosorbent-assay plate reader. The half maximal inhibitory concentration $\left(\mathrm{IC}_{50}\right)$ was determined by linear regression (Graph Pad Prism). ${ }^{11}$ The results were classified as: $\mathrm{IC}_{50} \leq 100 \mu \mathrm{g} \mathrm{mL}^{-1}$ was considered active, $\mathrm{IC}_{50}$ between 101 and $200 \mu \mathrm{g} \mathrm{mL}-1$ was considered moderate active, and $\mathrm{IC}_{50} \geq 200 \mu \mathrm{g} \mathrm{mL}^{-1}$ was considered to be inactive. ${ }^{12,13}$

Determination of the selectivity index (SI)

The selectivity index (SI) was determined by the following equation: ${ }^{14}$

$\mathrm{SI}=\frac{\mathrm{CC}_{50} \text { in cell }}{\mathrm{IC}_{50} \text { against parasite }}$

where $\mathrm{CC}_{50}$ is the $50 \%$ cytotoxicity concentration.

For the interpretation of the results of the selectivity index, it was considered that an SI greater than 1 indicates that the compound under study presents a greater toxicity to the parasite than to the macrophage. An SI less than 1 indicates that the compound has a higher toxicity to the macrophage than to the parasite. The higher the numerical value of SI, the more selective the compound under study, that is, less toxic to the macrophage and active for the parasite. $^{14}$

\section{Computational methodology}

\section{Molecular docking}

Molecular docking is one of the main methods of structure-based drug design (SBDD) molecular modeling, providing significant contributions in the discovery and optimization phases of leading compounds. ${ }^{15}$ Computational programs are used to perform calculations showing the best conformations of both ligands and receptors. This process consists of several steps, among them the choice of the receptor, binding site, evaluation of the intermolecular interactions and positioning of the ligand in the site.

The docking process was performed with the crystallographic protein of trypanothione reductase (TryR) from Leishmania infantum protein database (PDB) by means of the 2JK6 code, complexed with NADPH (nicotinamide adenine dinucleotide phosphate). In this step, the program AutoDock 4.2, ${ }^{16}$ using the Lamarckian genetic algorithm (LGA), was employed. ${ }^{17}$

The system was placed in a water box; its center was established in the structure of the NADPH, the box was adjusted with a dimension of $26 \times 24 \times 28$ points with a 
spacing of 0.375 , whose center of mass of the body was set at $\mathrm{X}=31.471, \mathrm{Y}=56.339$ and $\mathrm{Z}=-6.589$. The binder was treated with flexible simulations (all angles of freedom of the molecule were explored), generating 200 conformations for the compound with 270,000 generations in each run and the maximum number of energy evaluations was set at $25,000 .^{18}$

\section{Molecular dynamics}

In this study, the molecular dynamics simulation (MD) method was applied, which is an ideal method to analyze the conformational flexibility of protein molecules. ${ }^{19}$ The interactions between cytochalasin B and the crystallographic protein of trypanothione reductase (TryR) were analyzed.

\section{Results and Discussion}

Isolation and identification of cytochalasin B

The cytochalasin B (1) (Figure 1) was isolated and identified as described by us in a previous study. ${ }^{10}$

\section{Antipromastigote leishmanicidal activity}

Several classes of natural products have demonstrated activity against protozoa of Leishmania, such as alkaloids, terpenoids, lactones, lignans, flavonoids, coumarins, among others. ${ }^{20,21}$ Brissow et al..$^{22}$ reports the activity of cytochalasin $\mathrm{H}$ against promastigotes of L. amazonensis. Based on this information, the leishmanicidal assays with cytochalasin B (1) were performed. The cytochalasin B was assayed against the promastigote form of $L$. amazonensis and inhibited the growth with $\mathrm{IC}_{50}$ at $19.58 \mu \mathrm{g} \mathrm{mL}^{-1}$, also showed a $\mathrm{CC}_{50}$ of $74.36 \%$ for Vero cells and SI of 3.7977 , which shows that cytochalasin $\mathrm{B}$ is a potential drug-viable candidate against $L$. amazonensis promastigotes since it is toxic to the parasite and showed no appreciable toxicity to Vero cells.

Computational study of the leishmanicidal activity of cytochalasin B

\section{Molecular docking}

At the end of the docking simulations, free bond energy $(\mathrm{FBE})=-6.66 \mathrm{kcal} \mathrm{mol}^{-1}$ and inhibition constant $(\mathrm{IC})=13.10 \mu \mathrm{M}$ were obtained. The visualization of conformations, constructed by calculating molecular docking, was performed with the program Chimera 1.6. ${ }^{23}$ From these conformations, the one with the best score (FBE and IC) was chosen.

\section{Molecular dynamics}

The parameter files, topologies and coordinates necessary for the simulation of molecular dynamics were prepared, by which the stability through the root mean square deviation (RMSD) graph was observed in the system for a period of $100 \mathrm{~ns}$, according to Figure 2.

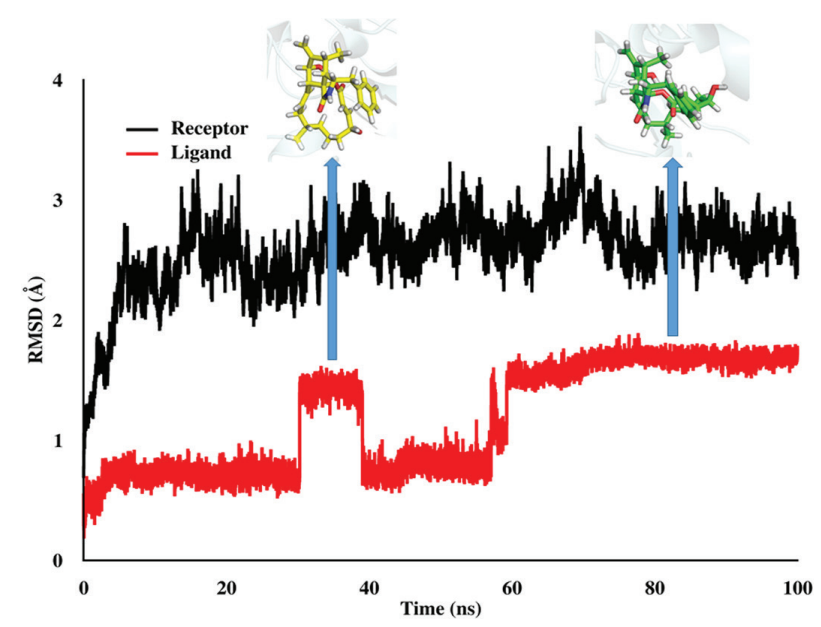

Figure 2. Molecular dynamics performed of the protein system with the ligand for $100 \mathrm{~ns}$.

Figure 2 shows that the crystallographic protein of trypanothione reductase, submitted to a molecular dynamics simulation, reached stability around $20 \mathrm{~ns}$, not observing significant fluctuations during the rest of the simulation time.

In the RMSD plot of the ligand, a conformational change in the ligand molecule is observed in approximately $30 \mathrm{~ns}$, which does not remain stable, returning to its initial state. At approximately $60 \mathrm{ns,} \mathrm{a} \mathrm{new} \mathrm{conformational} \mathrm{change}$ occurs, this time the ligand achieves a definitive stability. It is observed in the ligand structure that a conformational change occurred in the torsion angle between the atoms marked in Figures $3 \mathrm{a}$ and $3 \mathrm{~b}$.
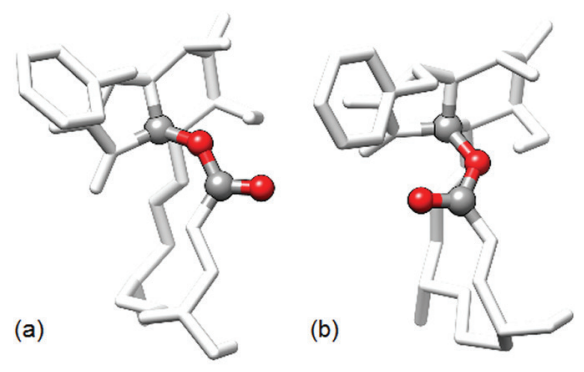

Figure 3. (a, b) Conformational change of the ligand.

This conformation contributes significantly to the stabilization of the system since it provides an interaction with the catalytic residue Thr51, where a hydrogen bonding of the $\mathrm{OH}$ group of Thr51 occurs with 


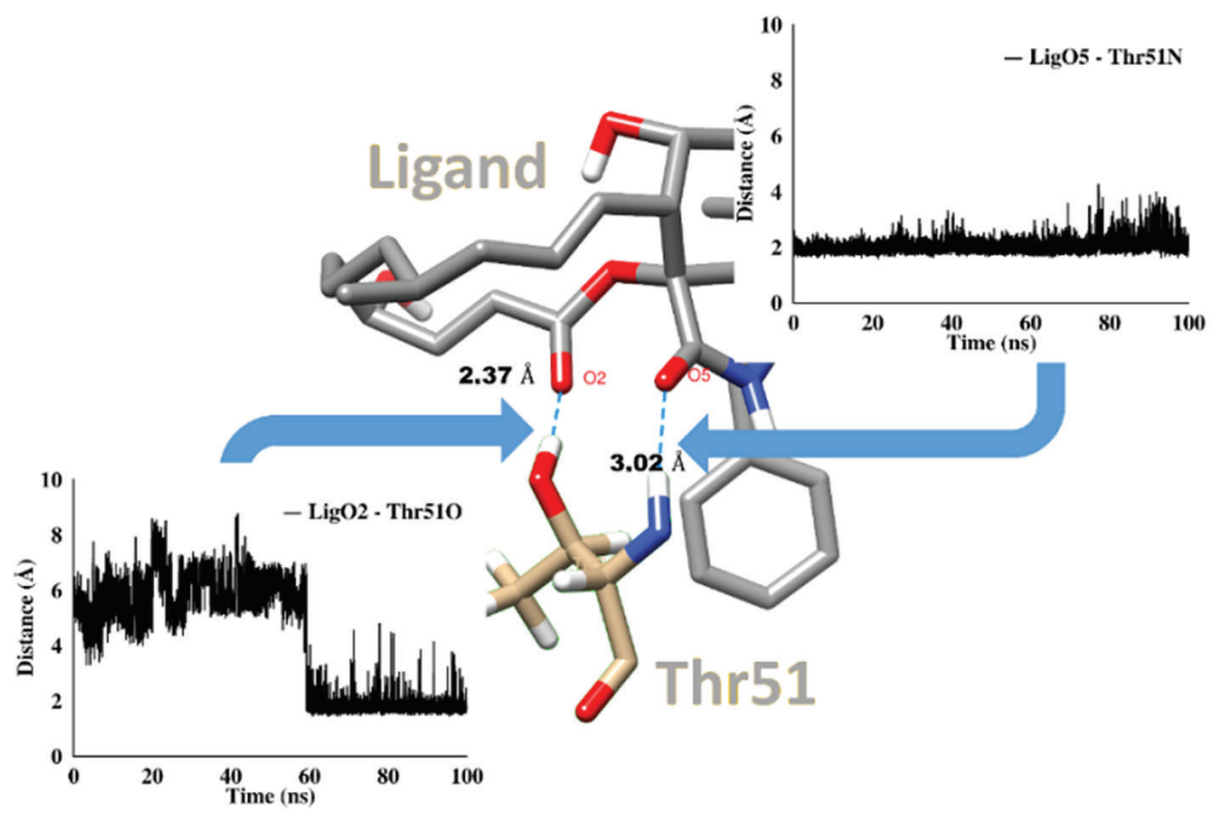

Figure 4. Interaction of Thr51 with the ligand during the $100 \mathrm{~ns}$ of simulation.

the carbonyl carbon $\left(\mathrm{O}_{2}\right)$ of the ligand (Figure 4). This interaction is established based on $60 \mathrm{~ns}$ of simulation and is important for the inhibition process, since it contributes to the binding of the ligand to the binding site. The interaction of the amino group of Thr51 with the oxygen $\mathrm{O} 5$ of the ligand is conserved throughout molecular dynamics, demonstrating that this residue is extremely important, as it performs two hydrogen bonds with the enzymatic receptor.

The estimate of bond free energies was calculated using the MMPBSA.py script. These energies were decomposed into contributions of individual residues, thus applying the so-called residue decomposition. In Figure 5, the Thr51 residue is shown to be the major contributor of cytochalasin B by binding to the active site along with Thr335 and Gly326 residues. This catalytic triad is largely responsible for the inhibitory process.

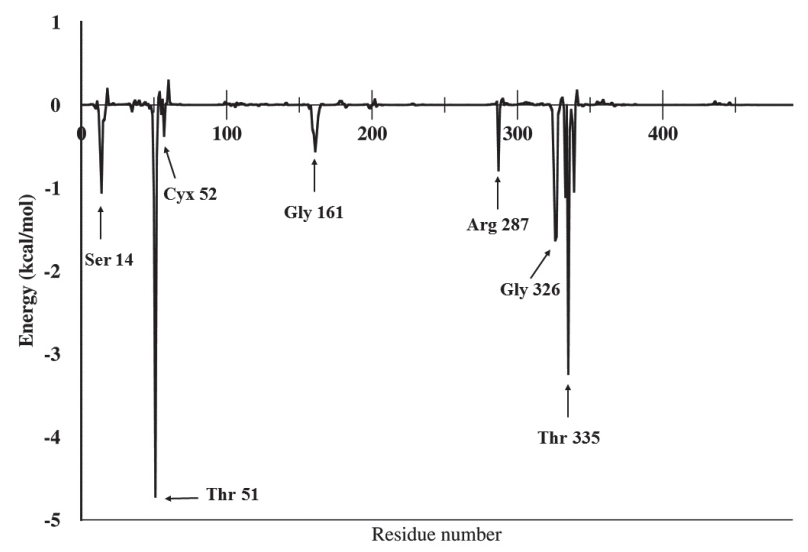

Figure 5. Residue interactions between the ligand and the receptor.
In a leishmanicidal study the trypanothione path has a great importance. This pathway is responsible for the oxidative control of parasite cells, which are sensitive to the increase of free radicals. Therefore, the control of these elements is important for the integrity and viability of the cells of these organisms, being TryR the enzyme with the highest activity in the control of oxidative stress, being an excellent target. ${ }^{24}$

Try $\mathrm{R}$ is a flavoprotein disulfide reductase dependent on NAPDH, and is found only in parasites of the Trypanosomatidae family. It is present in both $L$. infantum and $L$. amazonensis, thus enabling the use of the 2JK6 code protein in the study in question. This enzyme has the function of antioxidant; being the main form of control of reactive oxygen species (ROS) in these parasites, this protein replaces the glutathione reductase (GR) found in humans. ${ }^{25,26}$ The TryR is responsible for the reduction of the trypanothione disulfide $\left(\mathrm{T}[\mathrm{S}]_{2}\right)$ into its dithiol $\left(\mathrm{T}[\mathrm{SH}]_{2}\right)$, which in turn will reduce the oxidized tryparedoxin (TXN-1679 ${ }_{\text {ox }}$ ) into reduced tryparedoxin $\left(\mathrm{TXPN}_{\mathrm{red}}\right)$, and finally, it will reduce the oxidized tryparedoxin peroxidase $\left(\mathrm{TXPNx}_{\mathrm{ox}}\right)$ into reduced peroxidase tryparedoxin $\left(\mathrm{TXPNx}_{\text {red }}\right.$ ), which is capable of neutralizing ROS. The absence or deficiency of TryR causes the concentration of ROS within cells to increase, killing or impeding the growth of this protozoa. ${ }^{27}$

Thus, docking and MD studies are in agreement with the experimental results observed for the antipromastigote activity of cytochalasin B. They demonstrated that the inhibition of the parasite occurs due to the complexation of the substance with the crystal protein trypanothione reductase. 


\section{Conclusions}

The compound cytochalasin B (1) showed a good leishmanicidal activity against promastigotes of $L$. amazonensis. By the computational study, in order to investigate the potential interactions of Try $\mathrm{R}$ with the inhibitor cytochalasin $\mathrm{B}$, molecular docking and MD were performed. Through the investigation of the interactions of bonds of hydrogen and its distances, it was possible to conclude that the interaction of the amino group of Thr51 with the oxygen $\mathrm{O} 5$ of the ligand is conserved throughout molecular dynamics, demonstrating that this residue is extremely important, as it performs two hydrogen bonds with the enzymatic receptor. In terms of energy decomposition by the residue, there is a catalytic triad that manifests itself with the highest interaction energy: Thr51, Thr335 and Gly326. These residues combined guarantee the residence of the ligand at the receptor site, a fact observed throughout the time of molecular dynamics simulation. Thus, this study suggests that the activity observed of cytochalasin B against the promastigotes forms of $L$. amazonensis is possibly due to the inactivation of the enzyme trypanothione reductase.

\section{Supplementary Information}

Supplementary information is available free of charge at http://jbcs.sbq.org.br as a PDF file.

\section{Acknowledgments}

The authors thank the Fundação Amazônia de Amparo à Estudos e Pesquisas do Pará (FAPESPA), Conselho Nacional de Desenvolvimento Científico e Tecnológico (CNPq) and Coordenação de Aperfeiçoamento de Pessoal de Ensino Superior (CAPES) for financial support.

\section{References}

1. http://www.who.int/neglected_diseases/news/treating-morethan-one-billion-people-2017/en/, accessed on July 28, 2018.

2. Kealey, A.; Smith, R.; J. Health Care Poor Underserved 2010 , $21,53$.

3. Ruoti, M.; Oddone, R.; Lampert, N.; Orué, E.; Miles, M. A.; Alexander, N.; Rehman, A. M.; Njord, R.; Shu, S.; Brice, S.; Sinclair, B.; Krentel, A.; J. Trop. Med. 2013, 2013, article ID 538629.

4. Kaye, P.; Scott, P.; Nat. Rev. Microbiol. 2011, 9, 604.

5. Chakravarty, J.; Sundar, S.; J. Global Infect. Dis. 2010, 2, 167.

6. Sundar, S.; Olliaro, P. L.; Ther. Clin. Risk Manage. 2007, 3, 733.

7. Sant, A.; Carlos, M. R.; Rev. Virtual Quim. 2009, 1, 49.
8. Guido, R. V.; Andricopulo, A. D.; Oliva, G.; Estud. Av. 2010, 24,81 .

9. Pomel, S.; Rodrigo, J.; Hendra, F.; Cavé, C.; Loiseau, P. M.; Parasite 2012, 19, 63.

10. Feitosa, A. O.; Dias, A. C. S.; Ramos, G. C.; Bitencourt, H. R.; Siqueira, J. E. S.; Marinho, P. S. B.; Barison, A.; Ocampos, F. M. M.; Marinho, A. M. R.; Rev. Argent. Microbiol. 2016, 48, 259.

11. GraphPad Prism, version 5.04; GraphPad Software, La Jolla, CA, USA, 2010.

12. Mota, E. F.; Rosario, D. M.; Veiga, A. S. S.; Brasil, D. S. B.; Dolabela, M. F.; Pharmacogn. Mag. 2015, 11, 601.

13. Ngure, P. K.; Tonui, W. K.; Ingonga, J.; Mutai, C.; Kigondu, E.; Ng'ang'a, Z.; Rukunga, G.; Kimutai, A.; J. Med. Plants Res. 2009, 3, 61 .

14. Nakamura, C. V.; Santos, A. O.; Vendrametto, M. C.; Luize, P. S.; Dias Filho, B. P.; Cortez, D. A. G.; Ueda-Nakamura, T.; Braz. J. Pharmacogn. 2006, 16, 61.

15. Minini, L.; Álvarez, G.; González, M.; Cerecetto, H.; Merlino, A.; J. Mol. Graphics Modell. 2015, 58, 40.

16. Goodsell, D. S.; Morris, G. M.; Olson, A. J.; J. Mol. Recognit. 1996, $9,1$.

17. Morris, G. M.; Goodsell, D. S.; Halliday, R. S.; Huey, R.; Hart, W. E.; Belew, R. K.; Olson, A. J.; J. Comput. Chem. 1998, 19, 1639.

18. Costa, C. H. S.; Oliveira, A. R. S.; Pereira, M. J. S.; Figueiredo, A. F.; Ferreira, J. E. V.; Miranda, R. M.; J. Comput. Theor. Nanosci. 2015, 12, 3309.

19. Gao, Q.; Lu, C.; Wang, X.; Zhang, J.; Song, Y.; Xue, Y.; J. Mol. Model. 2018, 24, 95.

20. Bezerra, J. L.; Costa, G. C.; Lopes, T. C.; Carvalho, I. C. D. S.; Patrício, F. J.; Souza, S. M.; Amaral, F. M. M.; Rebelo, J. M. M.; Guerra, N. R. M.; Ribeiro, M. N. S.; Nascimento, F. R. F.; Braz. J. Pharmacogn. 2006, 16, 631.

21. Rocha, L. G.; Almeida, J. R. G. S.; Macedo, R. O.; BarbosaFilho, J. M.; Phytomedicine 2005, 12, 514.

22. Brissow, E. R.; Silva, I. P.; Siqueira, K. A.; Senabio, J. A.; Pimenta, L. P.; Januário, A. H.; Magalhães, L. G.; Furtado, R. G.; Tavares, D. C.; Sales, J. P. A.; Santos, J. L.; Soares, M. A.; Parasitol. Res. 2017, 116, 1823.

23. Pettersen, E. F.; Goddart, T. D.; Huang, C. C.; Cough, G. S.; Greenblatt, D. M.; Meng, E. C.; Ferrin, T. E.; J. Comput. Chem. 2004, 25, 1605.

24. Chawla, B.; Madhubala, R.; J. Parasit. Dis. 2010, 34, 1.

25. Fairlamb, A. H.; Cerami, A.; Annu. Rev. Microbiol. 1992, 46, 695.

26. Müller, S.; Liebau, E.; Walter, R. D.; Krauth-Siegel, L.; Trends Parasitol. 2003, 19, 320.

27. Kumar, S.; Ali, Md. R.; Bawa, S.; J. Pharm. BioAllied Sci. 2014, $6,222$.

Submitted: August 9, 2018 Published online: November 9, 2018 